\title{
Conformal transformations of leading twist operators in QCD: nonsinglet operators
}

\author{
A.N. Manashov* \\ Hamburg University \\ E-mail: alexander.manashov@desy.de
}

QCD evolution equations in minimal subtraction schemes have a hidden symmetry: the evolution kernel commutes with three operators that obey the commutation relations of the $S L(2)$ algebra. The explicit form of these operators can be found by analysis of QCD at the critical point in non-integer $d=4-2 \varepsilon$ space-time dimensions. Quantum corrections to symmetry generators in $d=4-2 \varepsilon$ correspond to the conformal symmetry breaking in the physical $(d=4)$ theory. The $S L$ (2) commutation relations lead to nontrivial constraints on the evolution kernels. We present the explicit two loop expressions for the generators of the $S L(2)$ algebra. This result allows one to restore the nonforward evolution kernels for the nonsinglet operators from the known NNLO anomalous dimensions.

Loops and Legs in Quantum Field Theory

24-29 April 2016

Leipzig, Germany

${ }^{*}$ Speaker. 


\section{Introduction}

Recent progress in accelerator and detector technologies has made it possible the study of hard exclusive reactions with identified particles in the final state. The relevant nonperturbative input in such processes involves operator matrix elements between states with different momenta, dubbed generalized parton distributions (GPDs), or vacuum-to-hadron matrix elements - the distribution amplitudes (DAs). The scale dependence of these matrix elements is governed by the renormalization group equations (RGE). The different momenta in the initial and the final state complicates the RG analysis since in this case one has to keep under control the mixing with operators involving total derivatives. The matrix of anomalous dimensions (for a given moment) is triangular: the diagonal entries correspond to the anomalous dimensions that are known to NNLO accuracy [1,2], but the nondiagonal contributions require a dedicated calculation.

A direct calculation of nondiagonal matrix elements in higher orders is quite challenging. However, it has been known for some time [3] that conformal symmetry of the QCD Lagrangian allows one to restore nondiagonal entries in the mixing matrix at given order of perturbation theory performing an additional calculation at one order less. This result was used to compute two-loop evolution kernels for the twist-two operators in QCD [4, 5, 6, 7, 8, 9].

An alternative technique was suggested in [10]. It employs the fact that the majority of QFT models in $d=4-2 \varepsilon$ dimensions possess a nontrivial Wilson-Fisher fixed point. At this (critical) point a theory enjoys, as a rule, exact scale and conformal invariance (see Ref. [11] for a review). Therefore one can expect that the RG kernels will commute with the generators of the conformal group. Of course, the generators differ from their canonical expression due to quantum corrections. Finally, taking into account that in the MS-like scheme the RG kernels (anomalous dimensions matrices) do not depend explicitly on the space-time dimension one concludes that the RG kernels are essentially the same in $d=4$ theory and $d=4-2 \varepsilon$ theory at the critical point ${ }^{1}$.

The utility of this approach was illustrated in [10] on several examples to two- and three-loop accuracy for scalar theories, and in [12] on the example of the two-loop evolution equation for flavor-nonsinglet operators in QCD. The two-loop expression for the generator of special conformal transformations was derived in Ref. [13]. Below we discuss the details of calculation and present the results.

\section{Conformal QCD}

QCD considered in $d=4-2 \varepsilon$ dimension possesses a nontrivial critical point for a large number of quark flavors. Indeed, the $\beta$-function

$$
\beta(a)=M \frac{d a}{d M}=2 a\left(-\varepsilon-\gamma_{g}\right),
$$

where $a=\alpha_{s} / 4 \pi$ and

$$
\gamma_{g}=\beta_{0} a+\beta_{1} a^{2}+\mathscr{O}\left(a^{3}\right)
$$

\footnotetext{
${ }^{1}$ At least for the models with one coupling constant.
} 
with

$$
\beta_{0}=\frac{11}{3} N_{c}-\frac{2}{3} N_{f}, \quad \beta_{1}=\frac{2}{3}\left[17 N_{c}^{2}-5 N_{c} N_{f}-3 C_{F} N_{f}\right]
$$

vanishes $\left(\beta\left(a_{*}\right)=0\right)$ for

$$
a_{*}(\varepsilon)=-\frac{\varepsilon}{\beta_{0}}-\left(\frac{\varepsilon}{\beta_{0}}\right)^{2} \frac{\beta_{1}}{\beta_{0}}+O\left(\varepsilon^{3}\right)
$$

The vanishing of the $\beta$-function implies that the correlators of local gauge-invariant operators transform in a proper way under scale and conformal transformations.

Due to operator mixing the RGEs for a set of the leading twist operators of a given dimension $\left\{\mathscr{O}_{i}, i=1, \ldots, n\right\}$, take the matrix form

$$
\left(\left(M \partial_{M}+\beta(a) \partial_{a}\right) \delta_{i k}+\gamma_{i k}(a)\right) \mathscr{O}_{i}=0
$$

where $\gamma_{i k}$ is the anomalous dimension matrix. The form of the matrix depends strongly on the choice of the basis operators, $\mathscr{O}_{i}$, and the symmetry properties of the problem are not transparent in this formulation. It proves more convenient to consider the generating function for the localoperators, the so-called light-ray operator [14]. For the twist-two nonsinglet operators we are interested in it takes the form

$$
[\mathscr{O}]\left(x ; z_{1}, z_{2}\right)=Z \mathscr{O}\left(x ; z_{1}, z_{2}\right)=Z \bar{q}\left(x+z_{1} n\right) h q\left(x+z_{2} n\right)=\sum_{m, k} \frac{z_{1}^{m} z_{2}^{k}}{m ! k !}\left[\bar{q}(x) \stackrel{\leftarrow}{D_{+}{ }^{m}} h \vec{h}_{+}^{k} q(x)\right],
$$

where $D_{+}=n^{\mu} D_{\mu}$ and the Wilson line is implied between the quark fields on the light-cone. The square brackets denote the renormalized operator in the $\overline{\mathrm{MS}}$ scheme and the renormalization factor $Z$ is an integral operator acting on the light-cone coordinates of the fields $z_{1}, z_{2}$

$$
Z=1+\sum_{k=0}^{\infty} \frac{1}{\varepsilon^{k}} Z_{k}(a), \quad Z_{k}(a)=\sum_{\ell=k}^{\infty} a^{\ell} Z_{k}^{(\ell)} .
$$

The RGE for the light-ray operator $[\mathscr{O}]$ takes the form

$$
\left(M \partial_{M}+\beta(a) \partial_{a}+\mathbb{H}(a)\right)[\mathscr{O}]\left(x ; z_{1}, z_{2}\right)=0,
$$

where $\mathbb{H}$ is an integral operator related to the renormalization factor (2.7) as follows

$$
\mathbb{H}(a)=2 \gamma_{q}(a)-M \frac{d}{d M} Z Z^{-1}=2 \gamma_{q}(a)+2 \sum_{\ell=1}^{\infty} \ell a^{\ell} Z_{1}^{(\ell)}=\sum_{k \geq 1} a^{k} \mathbb{H}^{(k)}
$$

$\gamma_{q}$ is the quark anomalous dimension. The evolution kernel can be written as [14]

$$
\mathbb{H}(a)[\mathscr{O}]\left(z_{1}, z_{2}\right)=\int_{0}^{1} d \alpha \int_{0}^{1} d \beta h(\alpha, \beta)[\mathscr{O}]\left(z_{12}^{\alpha}, z_{21}^{\beta}\right),
$$

where $z_{12}^{\alpha}=z_{1} \bar{\alpha}+z_{2} \alpha$ and $\bar{\alpha}=1-\alpha$. The weight function $h(\alpha, \beta)$ is given by a series in the coupling constant

$$
h(\alpha, \beta)=a h^{(1)}(\alpha, \beta)+a^{2} h^{(2)}(\alpha, \beta)+\ldots
$$


Let us stress here that the fixed-order kernels $h^{(k)}(\alpha, \beta)$ in the $\overline{\mathrm{MS}}$ scheme do not depend on the space-time dimension by construction. It means that the evolution kernel $\mathbb{H}$ at the critical point depends on the space-time dimension $d$ only through the value of the critical coupling, $a_{*}=a_{*}(\varepsilon)$,

$$
\mathbb{H}\left(a_{*}\right)=a_{*} \mathbb{H}^{(1)}+a_{*}^{2} \mathbb{H}^{(2)}+\ldots
$$

Thus the evolution kernel in the four dimensional theory inherits all symmetries of the kernel in the conformal $d$ dimensional theory.

\section{Symmetries}

It is natural to expect that the evolution kernel $\mathbb{H}\left(a_{*}\right)$ commutes with the generators of the conformal group. Most of the generators, however, act trivially, $\left[G_{\alpha}, \mathscr{O}\right]=0$, on the twist-two operators considered here leaving us with the so-called soft-collinear $\operatorname{SL}(2, R)$ subgroup of the conformal group. At the tree level the generators, $S_{\alpha}=\left\{S_{+}, S_{0}, S_{-}\right\}$are given by well known expressions

$$
S_{+}^{(0)}=z_{1}^{2} \partial_{z_{1}}+z_{2}^{2} \partial_{z_{2}}+2\left(z_{1}+z_{2}\right), \quad S_{0}^{(0)}=z_{1} \partial_{z_{1}}+z_{2} \partial_{z_{2}}+2, \quad S_{-}^{(0)}=-\partial_{z_{1}}-\partial_{z_{2}} .
$$

Beyond the leading order the generators are defined as follows. Let us expand the light-ray operators over the set of local conformal operators $\mathscr{O}_{N}$ and their descendants $\partial_{+}^{k} \mathscr{O}_{N}$,

$$
[\mathscr{O}]\left(x, z_{1}, z_{2}\right)=\sum_{N \geq 0} \sum_{k \geq 0} \Psi_{N k}\left(z_{1}, z_{2}\right) \partial_{+}^{k} \mathscr{O}_{N}(x) .
$$

The expansion coefficients (coefficient functions) $\Psi_{N k}\left(z_{1}, z_{2}\right)$ are homogeneous polynomials in $z_{1}, z_{2}$ of degree $N+k$ (of course they depend on the coupling constant $a_{*}$ or the space-time dimension $d$ ). The transformation properties of the conformal operator $\mathscr{O}_{N}(x)$ is determined entirely by its scaling dimension $\Delta_{N}^{*}=d+N-1+\gamma_{N}(a *)$ and spin $s_{N}=N+1$. Namely,

$$
i\left[\mathbf{D},\left[\mathscr{O}_{N}\right](x)\right]=\left(x \partial_{x}+\Delta_{N}^{*}\right)\left[\mathscr{O}_{N}\right](x) .
$$

for the scale and

$$
i\left[\mathbf{K}^{\mu},\left[\mathscr{O}_{N}\right](x)\right]=\left[2 x^{\mu}(x \partial)-x^{2} \partial^{\mu}+2 \Delta_{N}^{*} x^{\mu}+2 x^{v}\left(n^{\mu} \frac{\partial}{\partial n^{v}}-n_{v} \frac{\partial}{\partial n_{\mu}}\right)\right]\left[\mathscr{O}_{N}\right](x)
$$

for the special conformal transformations. The variation $S_{+}[\mathscr{O}]$ of the light-ray operator under the special conformal transformation $\mathbf{L}_{-}=\frac{i}{2} \bar{n}^{\mu} \mathbf{K}_{\mu}$ is defined as

$$
\begin{aligned}
{\left[\mathbf{L}_{-},[\mathscr{O}]\left(x, z_{1}, z_{2}\right)\right] } & =\sum_{N \geq 0} \sum_{k \geq 0} \Psi_{N k}\left(z_{1}, z_{2}\right) \partial_{+}^{k}\left[\mathbf{L}_{-}, \mathscr{O}_{N}(x)\right] \\
& =\sum_{N \geq 0} \sum_{k \geq 0} \Psi_{N k}^{\prime}\left(z_{1}, z_{2}\right) \partial_{+}^{k} \mathscr{O}_{N}(x)=S_{+}[\mathscr{O}]\left(x, z_{1}, z_{2}\right)
\end{aligned}
$$

Going from the first to the second line we take into account that the conformal operators and their descendants form a basis in the space of local operators so that the expression $\partial_{+}^{k}\left[\mathbf{L}_{-}, \mathscr{O}_{N}(x)\right]$ can be re-expanded over the basis operators with a modified coefficient functions $\Psi_{N k}^{\prime}$. The other 
generators $S_{-}, S_{0}$ are defined in the same way. Such a definition of the generators $S_{ \pm, 0}$ is not explicit as it involves the functions $\Psi_{N k}$ which are not known. Nevertheless it guarantees that the generators $S_{\alpha}$ satisfy the $S L(2, R)$ commutation relations,

$$
\left[S_{+}, S_{-}\right]=2 S_{0} \quad\left[S_{0}, S_{ \pm}\right]= \pm S_{ \pm} .
$$

The commutation relations result in the following relations

$$
S_{-} \Psi_{N k}=-\Psi_{N k-1}, \quad S_{0} \Psi_{N k}=\left(j_{N}+k\right) \Psi_{N k}, \quad S_{+} \Psi_{N k}=(k+1)\left(2 j_{N}+k\right) \Psi_{N k+1},
$$

where the conformal spin $j_{N}=\left(\Delta_{N}^{*}+s_{N}\right) / 2$. Since the generator $S_{-}$corresponds to the translations it does not receive corrections and is given by its classical expression, $S_{-}=S_{-}^{(0)}$. It follows from the RG equation that the coefficient functions $\Psi_{N k}$ are the eigenfunctions of the evolution kernel, $\mathbb{H}\left(a_{*}\right) \Psi_{N k}=\gamma_{N}\left(a_{*}\right) \Psi_{N k}$. This leads immediately to the following expression for the generator $S_{0}$ :

$$
S_{0}=S_{0}^{(0)}+\gamma_{g}\left(a_{*}\right)+\frac{1}{2} \mathbb{H}\left(a_{*}\right) .
$$

The generator $S_{+}$can be presented as

$$
S_{+}=S_{+}^{(0)}+\left(z_{1}+z_{2}\right)\left(\gamma_{g}\left(a_{*}\right)+\frac{1}{2} \mathbb{H}\left(a_{*}\right)\right)+\left(z_{1}-z_{2}\right) \Delta_{+}\left(a_{*}\right),
$$

where the kernel $\Delta_{+}\left(a_{*}\right)$ commutes with $S_{-}$and $S_{0}^{(0)}$ and is usually referred to as conformal anomaly. Let us stress here that in distinction to the scaling (anomalous) dimensions at the critical point, which are physical observables and therefore do not depend on scheme, the evolution kernel, $\mathbb{H}\left(a_{*}\right)$, conformal anomaly $\Delta_{+}\left(a_{*}\right)$ and the coefficient functions $\Psi_{N k}$ are scheme-dependent. Their explicit form cannot be fixed from the symmetry consideration only and requires a separate calculation. The kernel $\mathbb{H}\left(a_{*}\right)$ and the conformal anomaly $\Delta_{+}\left(a_{*}\right)$ are, nevertheless, not independent. It follows from the commutation relations (3.6) that, as was expected, the kernel $\mathbb{H}\left(a_{*}\right)$ commutes with the generators $S_{\alpha}$. Two commutation relation, $\left[S_{-}, \mathbb{H}\right]=\left[S_{0}, \mathbb{H}\right]=0$, are satisfied by construction, while the last one, $\left[S_{+}, \mathbb{H}\left(a_{*}\right)\right]=0$, results in a nontrivial relation between $\mathbb{H}$ and $\Delta_{+}$. Namely,

$$
\left[S_{+}^{(0)}, \mathbb{H}\left(a_{*}\right)\right]=\left[\mathbb{H}\left(a_{*}\right), z_{1}+z_{2}\right]\left(\gamma_{g}\left(a_{*}\right)+\frac{1}{2} \mathbb{H}\left(a_{*}\right)\right)+\left[\mathbb{H}\left(a_{*}\right),\left(z_{1}-z_{2}\right) \Delta_{+}\left(a_{*}\right)\right] .
$$

Expanding Eq. (3.10) in a series in $a_{*}$ one gets

$$
\begin{aligned}
& {\left[S_{+}^{(0)}, \mathbb{H}^{(1)}\right]=0,} \\
& {\left[S_{+}^{(0)}, \mathbb{H}^{(2)}\right]=\left[\mathbb{H}^{(1)}, z_{1}+z_{2}\right]\left(\gamma_{g}^{(1)}+\frac{1}{2} \mathbb{H}^{(1)}\right)+\left[\mathbb{H}^{(1)},\left(z_{1}-z_{2}\right) \Delta_{+}^{(1)}\right],}
\end{aligned}
$$

and so on. Since the kernel $\mathbb{H}\left(a_{*}\right)$ commutes with $S_{-}^{(0)}$ and $S_{0}^{(0)}$ these equations demonstrate that the non-invariant part of the $\ell$-loop kernel (with respect to the canonical conformal transformations) is completely fixed by lower order kernels $\mathbb{H}^{(k)}, \Delta_{+}^{(k)}, k=1, \ldots, \ell-1$. 


\section{Conformal Ward Identity}

An explicit form of the generator of special conformal transformations $S_{+}$, or the conformal anomaly $\Delta_{+}\left(a_{*}\right)$ can be derived from analysis of the conformal Ward Identity (CWI) [7, 9, 13]. It follows from the definition (3.5) of the generator $S_{+}$that the correlator of the two light-ray operators,

$$
\mathscr{G}(x ; z, w)=\left\langle\left[\mathscr{O}^{(n)}\right](0, z)\left[\mathscr{O}^{(\bar{n})}\right](x, w)\right\rangle=\mathscr{N} \int D \Phi e^{-S_{R}(\Phi)}\left[\mathscr{O}^{(n)}\right](0, z)\left[\mathscr{O}^{(\bar{n})}\right](x, w),
$$

satisfies the following relation

$$
\begin{aligned}
\frac{i}{2} \bar{n}^{\mu}\left(\left\langle\left[\mathbf{K}_{\mu},\left[\mathscr{O}^{(n)}\right](0, z)\right]\left[\mathscr{O}^{(\bar{n})}\right](x, w)\right\rangle+\left\langle\left[\mathscr{O}^{(n)}\right](0, z)\left[\mathbf{K}_{\mu},\left[\mathscr{O}^{(\bar{n})}\right](x, w)\right]\right\rangle\right)= \\
=\left[(n \bar{n}) S_{+}^{(z)}-\frac{1}{2} x^{2}\left(\bar{n} \partial_{x}\right)\right] \mathscr{G}(x ; z, w)=0,
\end{aligned}
$$

where $z=\left\{z_{1}, z_{2}\right\}, w=\left\{w_{1}, w_{2}\right\}$ and we assume that $(x \cdot n)=(x \cdot \bar{n})=0$. Let us stress here that (4.2) is a consequence of Eqs. (3.4) and (3.5). On the other hand one can derive Eq. (4.2) from invariance of the path integral in (4.1) under the change of variables:

$$
\Phi \mapsto \Phi+\delta_{K}^{\mu} \Phi, \quad \delta_{K}^{\mu} \Phi=\left(2 x_{\mu}(x \partial)-x^{2} \partial_{\mu}+2 \Delta_{\Phi} x_{\mu}-2 x^{v} \Sigma_{\mu v}\right) \Phi(x),
$$

see e.g. Ref. [15]. $\Sigma_{\mu \nu}$ in (4.3) is the generator of spin rotations,

$$
\Sigma_{\mu \nu} c=\Sigma_{\mu \nu} \bar{c}=0, \quad \Sigma_{\mu \nu} q=\frac{i}{2} \sigma_{\mu \nu} q, \quad \Sigma_{\mu v} A_{\alpha}=g_{v \alpha} A_{\mu}-g_{\mu \alpha} A_{v}
$$

and it is convenient to choose the dimensions of the QCD fundamental fields, $\Delta_{\Phi}$, as follows [9]:

$$
\Delta_{q}=\frac{3}{2}-\varepsilon, \quad \Delta_{A}=1, \quad \Delta_{c}=0, \quad \Delta_{\bar{c}}=2-\varepsilon .
$$

The CWI takes the form

$$
\left\langle\delta\left[\mathscr{O}^{(n)}\right](0, z)\left[\mathscr{O}^{(\bar{n})}\right](x, w)\right\rangle+\left\langle\left[\mathscr{O}^{(n)}\right](0, z) \delta\left[\mathscr{O}^{(\bar{n})}\right](x, w)\right\rangle=\left\langle\delta S_{R}\left[\mathscr{O}^{(n)}\right](0, z)\left[\mathscr{O}^{(\bar{n})}\right](x, w)\right\rangle,
$$

where $\delta=\bar{n}_{\mu} \delta_{K}^{\mu}, \delta S_{R}$ is the corresponding variation of the QCD action (in Euclidean space)

$$
\delta_{K}^{\mu} S_{R}=\int d^{d} x 2 x^{\mu}\left(\mathscr{N}(x)-(d-2) \partial^{\rho} \mathscr{B}_{\rho}(x)\right),
$$

and

$$
\mathscr{N}(x)=2 \varepsilon \mathscr{L}_{R}^{Y M+g f}=2 \varepsilon\left(\frac{1}{4} Z_{A}^{2} F^{2}+\frac{1}{2 \xi}(\partial A)^{2}\right), \quad \mathscr{B}_{\rho}(x)=Z_{c}^{2} \bar{c} D^{\rho} c-\frac{1}{\xi} A^{\rho}(\partial A) .
$$

It should be noted that the variation (4.6) does not vanish even at $d=4$. However, this nonvanishing term being the BRST variation does not contribute to the correlators of gauge invariant operators. This implies that a correlator of the nonlocal operator with two (anti)quark fields, $\langle\mathscr{O} q \bar{q}\rangle$, is not a convenient object for analysis in the case of gauge theories. The further analysis goes along 
the lines of Ref. [16]. The operator $\mathscr{N}$ has to be written as the sum of renormalized (finite) operators,

$$
\mathscr{N}=-\frac{\beta(a)}{a}\left[\mathscr{L}^{Y M+g f}\right]-\left(\gamma_{A}+\gamma_{g}\right) \Omega_{A}-\sum_{\Phi \neq A} \gamma_{\Phi} \Omega_{\Phi}+\frac{\gamma_{A}}{\xi}\left[(\partial A)^{2}\right]+z_{c} \partial^{\mu} \Omega_{\mu}+z_{b} \partial_{\mu}\left[\mathscr{B}^{\mu}\right] .
$$

Here $\Omega_{\Phi}$ is an EOM operator, $\Omega_{\Phi}=\Phi(y)\left(\delta S_{R} / \delta \Phi(y)\right)$ and $\partial^{\mu} \Omega_{\mu}=\Omega_{\bar{c}}-\Omega_{c}, \gamma_{\Phi}$ are the field anomalous dimensions, and $z_{c}(g, \xi)$ and $z_{b}(g, \xi)$ are some finite coefficients. The last term being BRST variation does not contribute as well as the ghost EOM to the correlator (4.5). The other EOM terms together with the gauge fixing term result in the necessary modification of the scaling dimensions in the expression for the conformal generators. Nontrivial corrections to the $S_{+}$come from the first term on the r.h.s of (4.8). The correlator

$$
\left\langle\int d^{d} y \mathscr{N}(y)\left[\mathscr{O}^{(n)}\right](0, z)\left[\mathscr{O}^{(\bar{n})}\right](x, w)\right\rangle
$$

contains additional divergencies when the arguments of the renormalized operators coincide, i.e. when $y \sim 0$ or $y \sim x$ and gives nonzero contribution in spite of that it comes with vanishing coefficient $\beta\left(a_{*}\right)=0$. It can be shown that the correction to the generator $S_{+}$is related to the pair counterterms to the product of the operators $\mathscr{N}(y)\left[\mathscr{O}^{(n)}\right](0, z) \sim \delta(y) Z(\varepsilon)\left[\mathscr{O}^{(n)}\right](0, z)$. Technical details can be found in [13]. The one loop correction to the generator takes an extremely simple form $[7,12]$

$$
\Delta_{+}^{(1)} f\left(z_{1}, z_{2}\right)=2 C_{F} \int_{0}^{1} d \alpha \int_{0}^{1} d u \frac{\bar{\alpha}}{\alpha}\left[f\left(z_{12}^{\alpha u}, z_{2}\right)-f\left(z_{1}, z_{21}^{\alpha u}\right)\right]
$$

The two loop expression can be written [13] as

$$
\begin{aligned}
\Delta_{+}^{(2)} f\left(z_{1}, z_{2}\right)= & \int_{0}^{1} d \alpha \int_{0}^{\bar{\alpha}} d \beta\left[\omega(\alpha, \beta)+\omega^{\mathbb{P}}(\alpha, \beta) \mathbb{P}_{12}\right]\left[f\left(z_{12}^{\alpha}, z_{21}^{\beta}\right)-f\left(z_{12}^{\beta}, z_{21}^{\alpha}\right)\right] \\
& +\int_{0}^{1} d u \int_{0}^{1} d t \varkappa(t)\left[f\left(z_{12}^{u t}, z_{2}\right)-f\left(z_{1}, z_{21}^{u t}\right)\right]
\end{aligned}
$$

where $\mathbb{P}_{12} f\left(z_{1}, z_{2}\right)=f\left(z_{2}, z_{1}\right)$ is the permutation operator. The kernels $\varkappa(t), \omega(\alpha, \beta), \omega^{\mathbb{P}}(\alpha, \beta)$ receive contributions of three different color structures

$$
\begin{aligned}
\varkappa(t) & =C_{F}^{2} \varkappa_{F F}(t)+C_{F} C_{A} \varkappa_{F A}(t)+C_{F} \beta_{0} \varkappa_{b F}(t), \\
\omega(\alpha, \beta) & =C_{F}^{2} \omega_{F F}(\alpha, \beta)+C_{F} C_{A} \omega_{F A}(\alpha, \beta), \\
\omega^{\mathbb{P}}(\alpha, \beta) & =C_{F}^{2} \omega_{F F}^{\mathbb{P}}(\alpha, \beta)+C_{F} C_{A} \omega_{F A}^{\mathbb{P}}(\alpha, \beta) .
\end{aligned}
$$

As an example, we give here the result for the $C_{F} C_{A}$ color structure,

$$
\begin{aligned}
\varkappa_{F A}(t)= & \frac{2 \bar{t}}{t}\left\{(2+t)\left[\operatorname{Li}_{2}(\bar{t})-\operatorname{Li}_{2}(t)\right]-(2-t)\left(\frac{t}{\bar{t}} \ln t+\ln \bar{t}\right)-\frac{\pi^{2}}{6} t-\frac{4}{3}-\frac{t}{2}\left(1-\frac{t}{\bar{t}}\right)\right\} \\
\omega_{F A}(\alpha, \beta)= & 2\left[\left(\frac{1}{\alpha}-\alpha\right)\left[\operatorname{Li}_{2}\left(\frac{\beta}{\bar{\alpha}}\right)-\operatorname{Li}_{2}(\beta)-2 \operatorname{Li}_{2}(\alpha)-\ln \alpha \ln \bar{\alpha}\right]+\frac{\alpha}{\tau}(\tau \ln \tau+\bar{\tau} \ln \bar{\tau})\right. \\
& \left.-\bar{\beta} \ln \alpha-\frac{\bar{\alpha}}{\alpha} \ln \bar{\alpha}\right], \\
\omega_{F A}^{\mathbb{P}}(\alpha, \beta)= & 2\left[\left(\bar{\alpha}-\frac{1}{\bar{\alpha}}\right)\left[\operatorname{Li}_{2}\left(\frac{\alpha}{\bar{\beta}}\right)-\mathrm{Li}_{2}(\alpha)-\ln \bar{\alpha} \ln \bar{\beta}\right]+\alpha \bar{\tau} \ln \bar{\tau}+\frac{\beta^{2}}{\bar{\beta}} \ln \bar{\alpha}\right] .
\end{aligned}
$$

Here $\tau=\alpha \beta / \bar{\alpha} \bar{\beta}$. Explicit expressions for the other color structures can be found in Ref. [13]. 


\section{Acknowledgments}

The author thanks V.M. Braun, S. Moch and M. Strohmaier for the collaboration on this project. This work was supported by Deutsche Forschungsgemeinschaft (DFG) with the grant MO 1801/1-1.

\section{References}

[1] S. Moch, J. A. M. Vermaseren and A. Vogt, The Three loop splitting functions in QCD: The Nonsinglet case, Nucl. Phys. B 688 (2004) 101.

[2] A. Vogt, S. Moch and J. A. M. Vermaseren, The Three-loop splitting functions in QCD: The Singlet case, Nucl. Phys. B 691 (2004) 129.

[3] D. Müller, Constraints for anomalous dimensions of local light cone operators in $\phi^{3}$ in six-dimensions theory, Z. Phys. C 49 (1991) 293.

[4] D. Müller, Conformal constraints and the evolution of the nonsinglet meson distribution amplitude, Phys. Rev. D 49 (1994) 2525.

[5] D. Müller, Restricted conformal invariance in QCD and its predictive power for virtual two photon processes, Phys. Rev. D 58 (1998) 054005.

[6] A. V. Belitsky and D. Müller, Predictions from conformal algebra for the deeply virtual Compton scattering, Phys. Lett. B 417 (1998) 129.

[7] A. V. Belitsky and D. Müller, Next-to-leading order evolution of twist-2 conformal operators: The Abelian case, Nucl. Phys. B 527 (1998) 207.

[8] A. V. Belitsky, A. Freund and D. Müller, Evolution kernels of skewed parton distributions: Method and two loop results, Nucl. Phys. B 574 (2000) 347.

[9] A. V. Belitsky and D. Müller, Broken conformal invariance and spectrum of anomalous dimensions in QCD, Nucl. Phys. B 537 (1999) 397.

[10] V. M. Braun and A. N. Manashov, Evolution equations beyond one loop from conformal symmetry, Eur. Phys. J. C 73 (2013) 2544.

[11] Y. Nakayama, Scale invariance vs conformal invariance, Phys. Rept. 569 (2015) 1.

[12] V. M. Braun and A. N. Manashov, Two-loop evolution equations for light-ray operators, Phys. Lett. B 734 (2014) 137.

[13] V. M. Braun, A. N. Manashov, S. Moch and M. Strohmaier, Two-loop conformal generators for leading-twist operators in QCD, JHEP 1603 (2016) 142

[14] I. I. Balitsky and V. M. Braun, Evolution Equations for QCD String Operators, Nucl. Phys. B 311 (1989) 541.

[15] V. M. Braun, G. P. Korchemsky and D. Müller, The Uses of conformal symmetry in QCD, Prog. Part. Nucl. Phys. 51 (2003) 311.

[16] A. N. Vasil'ev, The field theoretic renormalization group in critical behavior theory and stochastic dynamics, Boca Raton, USA: Chapman \& Hall/CRC (2004) 681 p. 